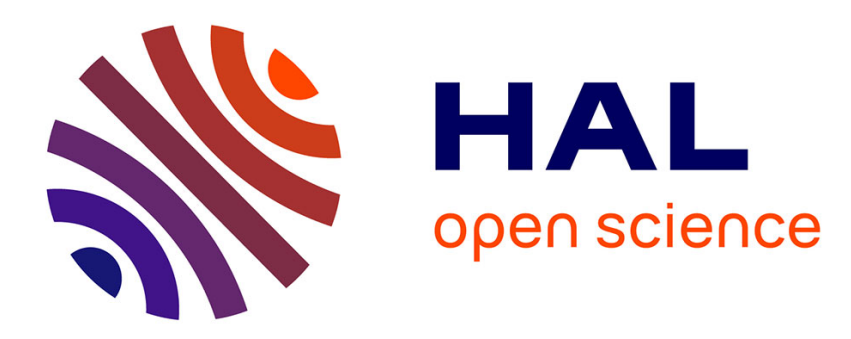

\title{
Caractérisation du rayonnement de la table d'harmonie d'un piano droit
}

Ph. Derogis, R. Caussé

\section{To cite this version:}

Ph. Derogis, R. Caussé. Caractérisation du rayonnement de la table d'harmonie d'un piano droit. Journal de Physique IV Proceedings, 1994, 04 (C5), pp.C5-609-C5-612. 10.1051/jp4:19945130 jpa00252807

\section{HAL Id: jpa-00252807 https://hal.science/jpa-00252807}

Submitted on 1 Jan 1994

HAL is a multi-disciplinary open access archive for the deposit and dissemination of scientific research documents, whether they are published or not. The documents may come from teaching and research institutions in France or abroad, or from public or private research centers.
L'archive ouverte pluridisciplinaire HAL, est destinée au dépôt et à la diffusion de documents scientifiques de niveau recherche, publiés ou non, émanant des établissements d'enseignement et de recherche français ou étrangers, des laboratoires publics ou privés. 


\title{
Caractérisation du rayonnement de la table d'harmonie d'un piano droit
}

\author{
Ph. DEROGIS et R. CAUSSÉ
}

I.R.C.A.M., 1 Place Igor Stravinsky, 75004 Paris, France

\begin{abstract}
In this paper we present a simple model of a upright piano soundboard as a baffled isotrope plate. This model enables us to calculate analytically the sound radiation of the soundboard in the back semi-infinite space of the piano and to find the frequency threshold of the appearance of the lobe of maximum pressure for each mode. These results are compared with measurements on a real upright piano. We find that the resonance frequency of the modes are less than this frequency threshold, therefore the maximum sound radiation can not occur. The pattern of the sound radiation appears to follow the theoretical model but more experiments will be required to show this clearly.
\end{abstract}

\section{Introduction}

Nous présentons dans cet article un modèle simple permettant d'estimer le rayonnement de la table d'harmonie d'un piano droit. Après un bref exposé de notre modèle therorique, nous discutons les différentes caractéristiques du champ acoustique prédit, puis, dans une deuxième partie, nous présentons les résultats des mesures effectúes et comparons ces résultats avec ceux prédits par la théorie.

\section{Le modèle}

Le piano, comme tous les instruments à cordes, rayonne de l'énergie grâce au couplage de ses cordes à la table d'harmonie par l'intermédiaire du chevalet. Les cordes ne rayonnant que très peu d'énergie de part leur faible surface, leur rayonnement a été négligé. II nous restait donc à modéliser la table d'harmonie montée dans son meuble. Nous l'avons approximée par une plaque rectangulaire isotrope supportée, bafflée par un plan infini. En effet, d'une part des études [1] [2] [3] ont montré que pour les premiers modes (basses fréquences) l'anisotropie du bois et la géométrie de la table ont peu d'effet, et d'autre part l'onde arrière générée par la table étant enfermée dans le meuble, nous avons supposé que la plaque ne rayonnait que par une seule face. Enfin, l'hypothèse du plan infini nous a permis de mener plus facilement à bien nos calculs en bénéficiant des résultats de la littérature [4] et [5].

\section{Rayonnement d'une plaque isotrope bafflée sur son mode $[m, n]$}

L'équation de propagation des ondes de flexion dans une plaque isotrope de longueur $a$, de largeur $b$, d'épaisseur $h$ et de masse volumique $\rho$ est la suivante:

$$
D \Delta^{2} z(x, y, \omega)-\rho h \omega^{2} z(x, y, \omega)=0 \quad \text { où } \quad D=\frac{E h^{3}}{12\left(1-v^{2}\right)}
$$

les fonctions propres $\left(\phi_{m n}\right)$ de cette équation vérifiant les conditions aux limites (plaque supportée) et leurs valeurs propres associées $\left(\omega_{m n}\right)$ sont telles que:

$$
\phi_{m n}(x, y)=\sin \left(\frac{m \Pi x}{a}\right) \sin \left(\frac{n \Pi y}{b}\right) \quad \text { et } \quad \omega_{m n}=\sqrt{\frac{D}{\rho h}}\left(\left(\frac{m \Pi}{a}\right)^{2}+\left(\frac{n \Pi}{b}\right)^{2}\right)
$$

Pour le mode $(m, n)$, l'accélération au point $\left(x_{0}, y_{0}\right)$ de la plaque est donnée par l'équation (2) et vaut:

$$
\gamma_{m n}\left(x_{0}, y_{0}\right)=\gamma_{m n} \sin \left(\frac{m \Pi x_{0}}{a}\right) \sin \left(\frac{n \Pi y_{0}}{b}\right)
$$

Le calcul de la pression par équation intégrale est alors possible et nous donne, avec les approximations du champ lointain: 


$$
|p(R, \theta, \Phi)|^{2}=\frac{4 \gamma_{m n}^{2}}{R^{2}} \times\left(\frac{a b \rho_{0}}{m n \Pi^{3}}\right)^{2} \times\left\{\frac{\left(\begin{array}{c}
\cos \\
\sin
\end{array}\right)\left(\frac{\lambda a}{2}\right) \times\left(\begin{array}{l}
\cos \\
\sin
\end{array}\right)\left(\frac{\mu b}{2}\right)}{\left[\left(\frac{\lambda a}{m \Pi}\right)^{2}-1\right] \times\left[\left(\frac{\mu b}{n \Pi}\right)^{2}-1\right]}\right\}^{2}
$$

les cosinus et les sinus étant utilisés respectivement quand $\mathrm{m}$ et $\mathrm{n}$ sont paires ou impaires. $\lambda$ et $\mu$ sont définis comme suit:

$$
\lambda=k \sin (\theta) \cos (\Phi) \quad \text { et } \quad \mu=k \sin (\theta) \sin (\Phi)
$$

A la figure 1 , nous présentons les figures de rayonnement prédites par l'équation (4) pour les modes [1,1], $[3,1]$, et $[2,2]$ auxquels nous nous sommes interessés lors de nos mesures.

\section{Figure 1 : Rayonnément théorique d'une plaque isotrope bafflée}

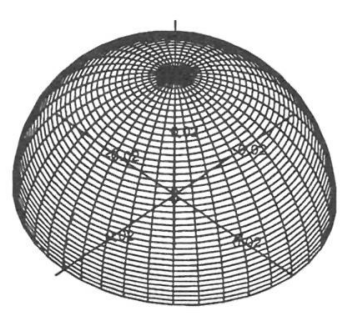

Rayonnement du mode $[1,1]$ a $90 \mathrm{~Hz}$

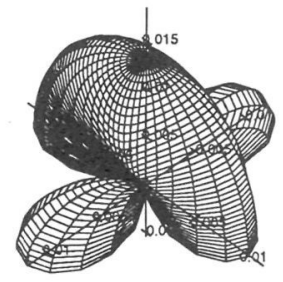

Rayonnement du mode $[3,1]$ à $184 \mathrm{~Hz}$

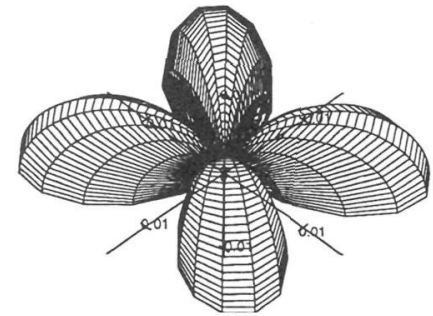

Rayonnement du mode [2,2] a $234 \mathrm{~Hz}$

On remarque à partir de l'équation (4), que pour une distance donnée, seuls les paramètres $\lambda$ et $\mu$ sont amenés à varier en fonction de $\theta$ et $\phi$ dans un intervalle dont les bornes sont fixés par la fréquence d'excitation de la plaque. Nous pouvons donc nous ramener à l'étude de la famille de fonctions:

$$
f_{m}(\lambda a)=\frac{\left(\begin{array}{l}
\cos \\
\sin
\end{array}\right)\left(\frac{\lambda a}{2}\right)}{\left[\left(\frac{\lambda a}{m \Pi}\right)^{2}-1\right]}
$$

Nous présentons en figure 2 trois de ces courbes. Deux remarques sont à faire. Premièrement, l'intervalle de variation de $\lambda$ étant fixé par la fréquence, plus elle sera élevée, plus le nombre de lobes sera important. Deuxièmement, pour tous les modes (excepté le mode [1,1]) il est nécessaire de dépasser une fréquence seuil pour que le lobe de maximum de pression apparaisse. On a:

$$
p_{m n_{\text {Max }}}=\gamma_{m n}\left(\frac{\rho_{0} a b}{8 \Pi R}\right) \quad \text { et } \quad f_{\text {seuil }}=\max \left(\frac{m c}{2 a}, \frac{n c}{2 b}\right)
$$

Ce maximum ne dépend que de la surface de la plaque et du maximum d'accélération. La fréquence, elle, correspond à la coïncidence entre la longueur d'onde dans la plaque et celle des ondes de pression dans l'air.

Figure 2 : Courbes représentatives des trois de fonctions $f_{m}$

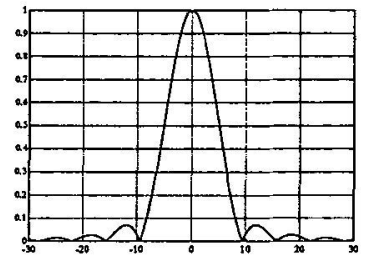

Courbe représentative de la fonction $f_{1}$

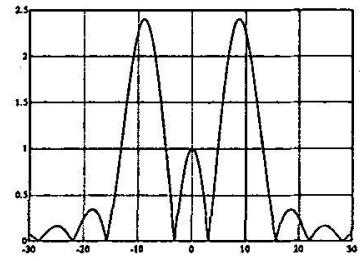

Courbe représentative de la fonction $f_{3}$

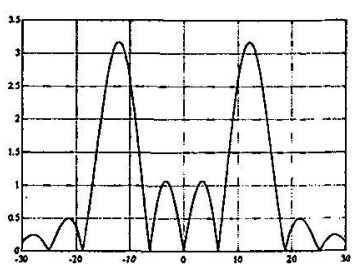

Courbe représentative de la fonction $f_{4}$ 


\section{Mesures et résultats}

Nous avons localisé les premiers modes de vibration de notre table d'harmonie grâce à deux méthodes. Premièrement, l'étude des pics communs de fonctions de mobilité mesurées par une méthode impulsionnelle (marteau d'impact) nous a permis de cerner les fréquences "intéressantes". Deuxièmement, la table étant excitée sinusoïdalement, des mesures d'accélération (en module et en phase) nous ont permis de trouver plus précisément les fréquences propres ainsi que les modes auxquels elles étaient attachées. Nous présentons dans le tableau 1 nos résultats ainsi que ceux obtenus par d'autres auteurs. Comme Nakamura [1] et Rossing [3] nous n'avons

Tableau 1: Modes et fréquences de vibration pour différents pianos droits (avec cordes)

\begin{tabular}{|c|c|c|c|c|}
\hline Mode & Yhamaha & Kimball & (Nakamura) & Schimmel \\
\hline \hline$[1,1]$ & $90 \mathrm{~Hz}$ & $117 \mathrm{~Hz}$ & $100 \mathrm{~Hz}$ & $103 \mathrm{~Hz}$ \\
\hline$[2,1]$ & $124 \mathrm{~Hz}$ & $132 \mathrm{~Hz}$ & - & - \\
\hline$[1,2]$ & $144 \mathrm{~Hz}$ & $177 \mathrm{~Hz}$ & - & - \\
\hline$[3,1]$ & $184 \mathrm{~Hz}$ & $185 \mathrm{~Hz}$ & $182 \mathrm{~Hz}$ & $140 \mathrm{~Hz}$ \\
\hline$[2,2]$ & $234 \mathrm{~Hz}$ & $225 \mathrm{~Hz}$ & $227 \mathrm{~Hz}$ & - \\
\hline$[3,2]$ & $278 \mathrm{~Hz}$ & $300 \mathrm{~Hz}$ & - & $181 \mathrm{~Hz}$ \\
\hline
\end{tabular}

i. ce mode a été mis en évidence sur les courbes de mobilité mais n'a pas pu être clairement identifié par la méthode des accéléromètres.

pas pu identifier clairement les modes $[2,1]$ et $[1,2]$. Ceci est d'autant plus regrettable que ces modes présentent des figures de rayonnement simples et auraient été d'une grande utilité pour tester notre modèle.

Pour les mesures de rayonnement, nous avons excité la table d'harmonie grâce à un pot vibrant placé au ventre inférieur droit du mode $[2,2]$. Cette position nous permettait, d'une part, d'exciter les modes qui nous intéressaient et, d'autre part, évitait la proximité du chevalet et des tasseaux. Nous avons mesure la pression acoustique dans le plan horizontal grâce à un microphone placé à l'extrémité d'un bras de $1,65 \mathrm{~m}$ dont la rotation était assurée par une table tournante. Nous avons également mesuré l'accélération maximum aux ventres des modes afin de pouvoir prédire non seulement la forme des lobes, mais également le niveau réel de pression acoustique prévu à $1,65 \mathrm{~m}$ de la source. Nous présentons dans le tableau 2 et sur les figures 3 et 4 les résultats de nos mesures comparés aux résultats prédits. Le tableau 2 nous montre que, dans les deux cas où la mesure a été possible, la pression acoustique maximum prédite est supérieure à la pression maximum mesurée. Nous pensons que ceci est du à l'onde arrière (tout de même présente) qui, aux basses fréquences, vient court-circuiter l'onde avant. Nous remarquons également que pour tous les modes identifiés la fréquence de résonance du mode est inférieure à la fréquence seuil prédite par l'équation (7). Ce qui ne permet pas au maximum de pression possible d'occurer.

Nous représentons sur les figures 3 et 4 le rayonnement des trois modes mesurés. Pour le mode $[1,1]$ le rayonnement est quasi monopolaire. On remarque que la théorie prédit assez bien le rayonnement malgré un offset de $8 \mathrm{~dB}$ qui s'accentue à 0 et 180 degrés à cause du phénomène de court-circuit non prévu par le modèle. Pour le mode [3,1], nous avons ajouté la courbe de rayonnement en champ proche calculée grâce à une intégration numérique de l'équation intégrale. En effet la distance entre le micro et la source était beaucoup trop faible pour satisfaire aux hypothèses du champ lointain. Nous remarquons que cette dernière courbe approche assez bien la courbe expérimentale (vu la simplicité du modèle). Pour le mode [2,2] nous ne présentons que la courbe expérimentale car la topologie de ce mode nous obligeait à mesurer la pression dans un plan faisant un angle de 45 degrés avec le plan horizontal, chose difficilement réalisable. Nous vérifions tout de même le court-circuit $(-20 \mathrm{~dB})$ pratiquement perpendiculaire à la table.

Figure 3 : Courbe de rayonnement du mode [1,1]

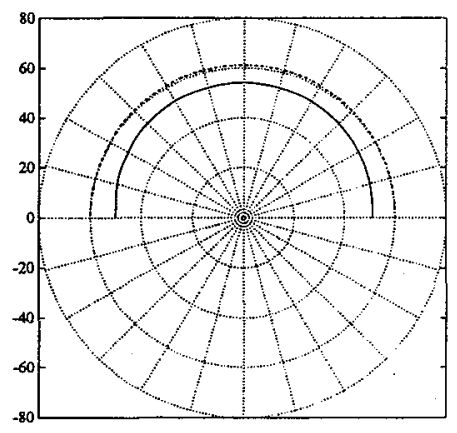

Courbe de rayonnement du mode $[1,1]$.

(-) Pression mesuree (en dB).

(-.) Pression calculee. 
Tableau 2: Bilan des mesures

\begin{tabular}{|c|c|c|c|c|c|c|}
\hline mode & fréquence & $\begin{array}{c}\text { accéération } \\
\left(\mathrm{en} \mathrm{ms}^{-2}\right)\end{array}$ & $\begin{array}{c}\text { Fréquence } \\
\text { seuil }\end{array}$ & $\begin{array}{c}\text { Pression max } \\
\text { mesurée }\end{array}$ & $\begin{array}{c}\text { Pression max } \\
\text { prédite }\end{array}$ & $\begin{array}{c}\text { Pression max } \\
\text { possible prédite }\end{array}$ \\
\hline \hline$[1,1]$ & $90 \mathrm{~Hz}$ & 0,1 & $0 \mathrm{~Hz}$ & $54 \mathrm{~dB}$ & $62 \mathrm{~dB}$ & $62 \mathrm{~dB}$ \\
\hline$[2,1]$ & $124 \mathrm{~Hz}$ & 1 & $251 \mathrm{~Hz}$ & - & $77 \mathrm{~dB}$ & $79 \mathrm{~dB}$ \\
\hline$[1,2]$ & $144 \mathrm{~Hz}$ & 1 & $386 \mathrm{~Hz}$ & - & $75 \mathrm{~dB}$ & $79 \mathrm{~dB}$ \\
\hline$[3,1]$ & $184 \mathrm{~Hz}$ & 0,8 & $376 \mathrm{~Hz}$ & $64 \mathrm{~dB}$ & $69 \mathrm{~dB}$ & $77 \mathrm{~dB}$ \\
\hline$[2,2]$ & $234 \mathrm{~Hz}$ & 1 & $386 \mathrm{~Hz}$ & - & $74 \mathrm{~dB}$ & $77 \mathrm{~dB}$ \\
\hline$[3,2]$ & $278 \mathrm{~Hz}$ & 1 & $386 \mathrm{~Hz}$ & - & $74 \mathrm{~dB}$ & $77 \mathrm{~dB}$ \\
\hline
\end{tabular}

Figure 4 : Courbes de rayonnement des modes $[3,1]$ et $[2,2]$

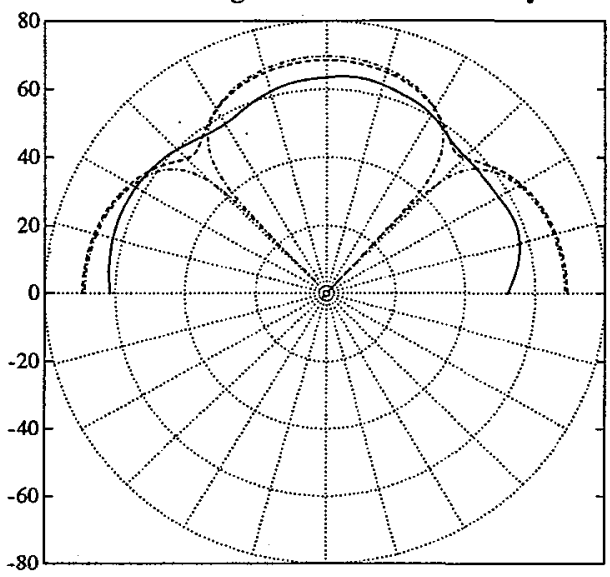

Courbes de rayonnement du mode $[3,1]$.

$(-)$ Pression mesurée (en dB),

(-.) Pression calculee en champ lointain.

(-) Pression calculée en champ proche.

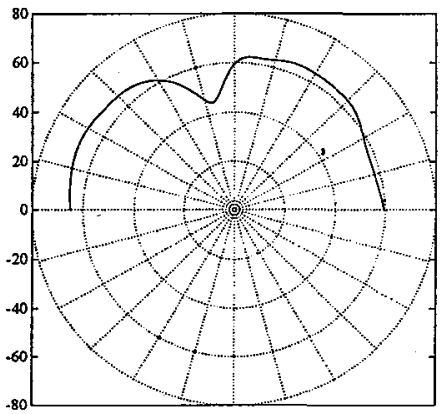

Courbe de rayonnement du mode [2,2].

$\rightarrow$ Pression mesurée (en $\mathrm{dB}$ ).

\section{Conclusion}

Le modèle de rayonnement que nous avons présenté, bien que très simple, nous permet de prévoir le rayonnement en champ lointain de la table d'harmonie d'un piano droit pour ses premiers modes dans le demi espace arrière. Nous avons mis en évidence que les dimensions de notre piano et les fréquences propres de la table sont telles que les lobes de rayonnement maximum ne peuvent pas apparaître, ce qui correspond à une perte d'efficacité de $9 \mathrm{~dB}$ au maximum. Pour les profils de rayonnement, les deux mesures comparatives semblent encourageantes. Il serait cependant nécessaire d'envisager d'autres mesures afin de pouvoir tester plus sérieusement ce modèle.

\section{Références}

[1] Nakamura I., The Vibrational character of the piano soundboard, Proc. 11th ICA Paris 385-388, (1983).

[2] Brelay H., Hoekje P., Rossing T.D., Vibrational modes and decay rates of solid and laminated piano soundboard, ISMA, Tokyo, 111-114, (1992).

[3] Rossing T.D., Wogram K. and Bork I., Vibration modes of a Piano Soundboard, (à paraître).

[4] Fahy F., Sound and structural Vibration, Academic Press, (1985).

[5] Lesueur C., Rayonnement acoustique des structures, Eyrolles, (1988). 